CUBO A Mathematical Journal

Vol.20, $N^{\underline{O}} 01$, (31-39). March 2018

\title{
Pre-regular sp-Open Sets in Topological Spaces
}

\author{
P. JeYanthi and P. NALAYini \\ Research Centre, Department of Mathematics, \\ Govindammal Aditanar College for Women, \\ Tiruchendur-628 215, Tamil Nadu, India. \\ T. NOIRI \\ Shiokita - cho, Hinagu, Yatsushiro - shi, \\ Kuvempu University \\ Kumamoto - ken, 869-5142 Japan \\ t.noiri@nifty.com
}

\begin{abstract}
In this paper, a new class of generalized open sets in a topological space, called preregular $s p$-open sets, is introduced and studied. This class is contained in the class of semi-preclopen sets and cotains all pre-clopen sets. We obtain decompositions of regular open sets by using pre-regular sp-open sets.
\end{abstract}

\section{RESUMEN}

En este artículo se introduce y estudia una nueva clase de conjuntos abiertos generalizados en un espacio topológico, llamados conjuntos pre-regulares sp-abiertos. Esa clase está contenida en la clase de conjuntos semi-preclopen y contiene todos los conjuntos pre-clopen. Obtenemos descomposiciones de conjuntos abiertos regulares usando conjuntos pre-regulares sp-abiertos.

Keywords and Phrases: Generalized open sets, preopen, regular open, pre-regular sp-open, decompositions of complete continuity.

2010 AMS Mathematics Subject Classification: 54A05. 


\section{Introduction}

In general topology, by repeated applications of interior (int) and closure (cl) operators several different new classes of sets are defined in the following way.

Definition 1. A subset $\mathrm{A}$ of a space $\mathrm{X}$ is said to be

i) semi-open [10] if $\mathrm{A} \subseteq \operatorname{cl}(\operatorname{int} A)$.

ii) preopen [11] if $\mathrm{A} \subseteq \operatorname{int}(\mathrm{clA})$.

iii) semi-preopen [2] or $\beta$-open [1] if $A \subseteq \operatorname{cl}(\operatorname{int}(\operatorname{clA}))$.

iv) $\alpha$-open [12] if $\mathrm{A} \subseteq \operatorname{int}(\operatorname{cl}(\operatorname{int} A))$.

v) regular open [13] if $\mathrm{A}=\operatorname{int}(\mathrm{clA})$.

vi) b-open [3] if $\mathrm{A} \subseteq \operatorname{cl}(\operatorname{int} A) \cup \operatorname{int}(\operatorname{cl} A)$.

vii) pre-regular $p$-open [9] if $\mathrm{A}=\operatorname{pint}(\mathrm{pclA})$.

The complements of the above open sets are called their respective closed sets.

Definition 2. A subset $\mathrm{A}$ of a space $\mathrm{X}$ is called a q-set [14] or $\delta$-set [5] if $\operatorname{int}(\operatorname{clA}) \subseteq \operatorname{cl}(\operatorname{int} A)$.

In this paper, we introduce and study a new class of sets, called pre-regular sp-open sets using pre-interior and semi-preclosure operators. This class is contained in the class of semi-preclopen sets and cotains all pre-clopen sets. Moreover, we investigate the relationship between this class of sets and other class of open sets. By using pre-regular sp-open sets, we obtain decompositions of regular open sets. In the last section, we obtain decompositions of complete continuity. Throughout this paper $(X, \tau)$ (briefly $X)$ denotes a topological space on which no separation axioms are assumed, unless explicity stated.

We recollect some of the relations that, together with their duals, we shall use in the sequel.

Proposition 1. [2] Let $\mathrm{A}$ be a subset of a space X. Then

i) $\operatorname{pcl} A=A \cup \operatorname{cl}(\operatorname{int} A)$ and $\operatorname{pint} A=A \cap \operatorname{int}(\operatorname{cl} A)$.

ii) $\operatorname{spcl} A=A \cup \operatorname{int}(\operatorname{cl}(\operatorname{int} A))$ and $\operatorname{spint} A=A \cap \operatorname{cl}(\operatorname{int}(\operatorname{cl} A))$.

iii) $\operatorname{pint}(\operatorname{spcl} A)=(A \cap \operatorname{int}(\operatorname{cl} A)) \cup \operatorname{int}(\operatorname{cl}(\operatorname{int} A))$.

iv) $\operatorname{pcl}(\operatorname{spint} A)=(A \cup \operatorname{cl}(\operatorname{int} A)) \cap \operatorname{cl}(\operatorname{int}(\operatorname{cl} A))$.

Definition 3. A function $\mathrm{f}: \mathrm{X} \rightarrow \mathrm{Y}$ is called completely continuous [4] (resp. $\alpha$-continuous [8],semi-continuous [10], q-continuous [14] ) if the inverse image of every open subset of $\mathrm{Y}$ is a regular open (resp. $\alpha$-open, semi-open, a q-set) subset of $\mathrm{X}$. 


\section{2 pre-regular $s p$-open sets}

In this section, we define and characterize pre-regular sp-open sets and study some of their properties.

Definition 4. A subset $\mathrm{A}$ of a topological space $(\mathrm{X}, \tau)$ is said to be pre-regular sp-open if $\mathrm{A}=$ pint(spclA). The complement of a pre-regular sp-open set is said to be pre-regular sp-closed.

We denote the collection of all pre-regular $s p$-open (resp. preopen,

preclosed, pre-semiopen, pre-semiclosed, pre-clopen, pre-semiclopen) sets of $(X, \tau)$ by $\operatorname{PRSPO}(X)$ (resp. $\mathrm{PO}(\mathrm{X}), \mathrm{PC}(\mathrm{X}), \mathrm{PSO}(\mathrm{X}), \operatorname{PSC}(\mathrm{X}), \mathrm{PCO}(\mathrm{X}), \mathrm{PSCO}(\mathrm{X})$ ).

Theorem 2.1. Let $(\mathrm{X}, \tau)$ be a topological space and $\mathrm{A}, \mathrm{B}$ subsets of $\mathrm{X}$. Then the following hold:

i) If $\mathrm{A} \subseteq \mathrm{B}$, then $\operatorname{pint}(\operatorname{spcl} \mathrm{A}) \subseteq \operatorname{pint}(\operatorname{spclB})$.

ii) If $\mathrm{A} \in \mathrm{PO}(\mathrm{X}, \tau)$, then $\mathrm{A} \subseteq \operatorname{pint}(\operatorname{spcl} \mathrm{A})$.

iii) If $\mathrm{A} \in \mathrm{SPC}(\mathrm{X}, \tau)$, then $\operatorname{pint}(\operatorname{spclA}) \subseteq \mathrm{A}$.

iv) We have $\operatorname{pint}(\operatorname{spcl}(\operatorname{pint}(\operatorname{spclA})))=\operatorname{pint}(\operatorname{spclA})$.

v) If $\mathrm{A} \in \mathrm{SPC}(\mathrm{X}, \tau)$, then pint $\mathrm{A}$ is a pre-regular sp-open set.

Proof. i) Suppose that $A \subseteq B$. Then $\operatorname{pint}(\operatorname{spcl} A) \subseteq \operatorname{pint}(\operatorname{spclB})$.

ii) Suppose that $A \in \operatorname{PO}(X, \tau)$. Since $A \subseteq \operatorname{spcl} A$, we have $A \subseteq \operatorname{pint}(\operatorname{spcl} A)$.

iii) Suppose that $A \in \operatorname{SPC}(X, \tau)$. Since pint $A \subseteq A$, we have $\operatorname{pint}(\operatorname{spcl} A) \subseteq A$.

iv) We have $\operatorname{pint}(\operatorname{spcl}(\operatorname{pint}(\operatorname{spcl} A))) \subset \operatorname{pint}(\operatorname{spcl}(\operatorname{spcl} A))=\operatorname{pint}(\operatorname{spcl} A)$ and $\operatorname{pint}(\operatorname{spcl}(\operatorname{pint}(\operatorname{spcl} A))) \supset$ $\operatorname{pint}(\operatorname{pint}(\operatorname{spcl} A))=\operatorname{pint}(\operatorname{spcl} A)$. Hence $\operatorname{pint}(\operatorname{spcl}(\operatorname{pint}(\operatorname{spcl} A)))=\operatorname{pint}(\operatorname{spcl} A)$.

v) Suppose that $A \in \operatorname{SPC}(X, \tau)$. By (i), we have $\operatorname{pint}(\operatorname{spcl}(\operatorname{pint} A)) \subseteq \operatorname{pint}(\operatorname{spcl} A)=\operatorname{pint} A$. On the other hand, we have pint $A \subseteq \operatorname{spcl}(\operatorname{pint} A)$. Therefore $\operatorname{pint} A \subseteq \operatorname{pint}(\operatorname{spcl}(\operatorname{pint} A))$ and hence $\operatorname{pint}(\operatorname{spcl}(\operatorname{pint} A))=\operatorname{pint} A$.

Remark 2.2. The family of pre-regular sp-open sets is not closed under finite union as well as finite intersection. It will be shown in the following example.

Example 2.3. Let $\mathrm{X}=\{\mathrm{a}, \mathrm{b}, \mathrm{c}, \mathrm{d}\}$ and $\tau=\{\emptyset,\{\mathrm{a}, \mathrm{b}\},\{\mathrm{a}, \mathrm{b}, \mathrm{c}\},\{\mathrm{a}, \mathrm{b}, \mathrm{d}\}, \mathrm{X}\}$. Then $\{\mathrm{a}\}$ and $\{\mathrm{b}\}$ are pre-regular sp-open sets but their union $\{\mathrm{a}, \mathrm{b}\}$ is not a pre-regular sp-open set. Moreover, $\{\mathrm{a}, \mathrm{c}, \mathrm{d}\}$ and $\{\mathrm{b}, \mathrm{c}, \mathrm{d}\}$ are pre-regular sp-open but their intersection $\{\mathrm{c}, \mathrm{d}\}$ is not a pre-regular sp-open set.

Theorem 2.5 and 2.6 give the characterizations of pre-regular sp-open sets. 
Theorem 2.4. Let $(\mathrm{X}, \tau)$ be a topological space. For a subset $\mathrm{A}$ of $\mathrm{X}$, the following are equivalent:

i) $\mathrm{A}$ is pre-regular sp-open.

ii) $\mathrm{A}=\operatorname{spclA} \cap \operatorname{int}(\mathrm{clA})$.

iii) $A=\operatorname{pint} A \cup \operatorname{int}(\operatorname{cl}(\operatorname{int} A))$.

Proof. It follows form Proposition 1.3.

Theorem 2.5. Let $(\mathrm{X}, \tau)$ be a topological space. A subset $\mathrm{A}$ of $\mathrm{X}$ is pre-regular sp-open if and only if it is preopen and semi-preclosed.

Proof. Let $A$ be pre-regular $s p$-open. Then $A=\operatorname{pint}(\operatorname{spcl} A)$. Hence $\operatorname{pint} A=\operatorname{pint}(\operatorname{pint}(\operatorname{spcl} A))=$ $\operatorname{pint}(\operatorname{spcl} A)=A$. Thus $A$ is preopen. By Theorem 2.5, $A=\operatorname{pint} A \cup \operatorname{int}(\operatorname{cl}(\operatorname{int} A))$ and $\operatorname{int}(\operatorname{cl}(\operatorname{int} A)) \subseteq A$. Therefore, $A$ is semi-preclosed. Conversely assume that $A$ is both preopen and semi-preclosed. Then $A=\operatorname{pint} A$ and $A=\operatorname{spcl} A$. Now pint $(\operatorname{spcl} A)=\operatorname{pint} A=A$. Hence $A$ is pre-regular $s p$-open.

Corolary 1. For a topological space $(\mathrm{X}, \tau)$, we have $\mathrm{PO}(\mathrm{X}) \cap \mathrm{PC}(\mathrm{X}) \subseteq \mathrm{PRSPO}(\mathrm{X}) \subseteq \mathrm{SPO}(\mathrm{X}) \cap$ $\operatorname{SPC}(X)$.

Proof. This is obvious.

Remark 2.6. The converse inclusions in Corollary 2.7 need not be true as the following examples show.

Example 2.7. Let $X=\{a, b, c, d\}$ and $\tau=\{\emptyset,\{a\},\{b\},\{a, b\},\{b, c\},\{a, b, c\}, X\}$.

Then $\{\mathrm{a}, \mathrm{d}\}$ is semi-preclopen but not pre-regular sp-open.

Example 2.8. Let $X=\{a, b, c, d\}$ and $\tau=\{\emptyset,\{a\},\{b\},\{c\},\{a, b\},\{a, c\},\{b, c\}$, $\{c, d\},\{a, c, d\},\{a, b, c\},\{b, c, d\}, X\}$. Then $\{c\}$ is pre-regular sp-open but it is not pre-clopen.

Theorem 2.9. In any space $(\mathrm{X}, \tau)$, the empty set is the only subset which is nowhere dense and pre-regular sp-open.

Proof. Suppose $A$ is nowhere dense and pre-regular $s p$-open. Then by Theorem 2.5, $A=\operatorname{pint}(\operatorname{spcl} A)=$ $\operatorname{spclA} \cap \operatorname{int}(\operatorname{clA})=\operatorname{spclA} \cap \emptyset=\emptyset$.

Remark 2.10. The notions of pre-regular sp-open sets and open sets (hence $\alpha$-open sets, semiopen sets, q-sets) are independent of each other. It is shown in [5] and [14] that every semi-open set is a q-set, that is, a $\delta$-set. 
Example 2.11. Let $X=\{a, b, c\}$ and $\tau=\{\emptyset,\{a, b\}, X\}$. Then $\{a, b\}$ is open hence $\alpha$-open, semiopen, a q-set but it is not pre-regular sp-open. Also, $\{\mathbf{a}\}$ is pre-regular sp-open but it is not a $q$-set.

Theorem 2.12. Every regular open set is pre-regular sp-open.

Proof. Let $A$ be regular open. Then $A=\operatorname{int}(\operatorname{cl} A)$. By Proposition 1.3, pint $(\operatorname{spcl} A)=(\operatorname{spcl} A) \cap$ $\operatorname{int}(\operatorname{cl}(\operatorname{spcl} A))=\operatorname{spcl} A \cap \operatorname{int}(\operatorname{cl}[A \cup \operatorname{int}(\operatorname{cl}(\operatorname{int} A))])=\operatorname{spcl} A \cap \operatorname{int}(\operatorname{cl} A)=\operatorname{spcl} A \cap A=A$. This shows that $A$ is pre-regular $s p$-open.

The above disscusion can be summarized in the following diagram:

\section{DIAGRAM}

regular open $\Rightarrow$ open $\Rightarrow \alpha$-open $\Rightarrow$ semi-open $\Rightarrow$ q-set

$\begin{array}{ccc}\Downarrow & \Downarrow & \Downarrow \\ \text { re-regular } s p \text {-open } \Rightarrow \text { preopen } \Rightarrow b \text {-open } \Rightarrow \text { semi-preopen }\end{array}$

Remark 2.13. A q-set and a semi-preopen set are independent by Example 2.13 and the following example.

Example 2.14. Let $\mathrm{R}$ be the real numbers with the usual topology. Then for each $\mathrm{x} \in \mathrm{R}$, $\operatorname{cl}(\operatorname{int}(\operatorname{cl}\{x\}))=\emptyset$ and it does not contain $\{x\}$. Hence $\{x\}$ is not semi-preopen. But $\operatorname{int}(\operatorname{cl}\{x\})=$ $\operatorname{cl}(\operatorname{int}\{x\})=\emptyset$ and $\{x\}$ is a q-set.

Theorem 2.15. Every pre-regular p-open set is pre-regular sp-open.

Proof. Let $A$ be pre-regular $p$-open. Then $A=\operatorname{pint}(\operatorname{pcl} A)$ and $A$ is preopen. Since spclA $\subseteq$ pclA, we have $\operatorname{pint}(\operatorname{spcl} A) \subseteq \operatorname{pint}(\operatorname{pcl} A)=A$. On the other hand, we have $A \subseteq \operatorname{spcl} A$. Since $A$ is preopen, $A=\operatorname{pint} A \subseteq \operatorname{pint}(\operatorname{spcl} A)$. Hence $A=\operatorname{pint}(\operatorname{spcl} A)$.

Theorem 2.16. For a subset $\mathrm{A}$ of a space $\mathrm{X}$, the following are equivalent:

i) A is regular open.

ii) $\mathrm{A}$ is pre-regular sp-open and a q-set.

iii) $\mathrm{A}$ is $\alpha$-open and semi-preclosed.

Proof. $i) \Rightarrow$ ii). Let $A$ be regular open. Then, by Theorem $2.14 \mathrm{~A}$ is pre-regular sp-open and also by Diagram, $A$ is a $q$-set.

ii) $\Rightarrow i)$. Since $A$ is a $q$-set, $\operatorname{int}(\operatorname{cl} A) \subset \operatorname{cl}(\operatorname{int} A)$ and $\operatorname{int}(\operatorname{cl} A) \subset \operatorname{int}(\operatorname{cl}(\operatorname{int} A))$

$\subset \operatorname{int}(\operatorname{cl} A)$. Therefore, we have int $(\operatorname{cl} A)=\operatorname{int}(\operatorname{cl}(\operatorname{int} A))$. By using Theorem 2.5, we obtain $\operatorname{int}(\operatorname{cl} A)=[A \cup \operatorname{int}(\operatorname{cl} A)] \cap \operatorname{int}(\operatorname{cl} A)=[A \cup \operatorname{int}(\operatorname{cl}(\operatorname{int} A))] \cap \operatorname{int}(\operatorname{cl} A)=\operatorname{spcl} A \cap \operatorname{int}(\operatorname{cl} A)=A$. i) $\Rightarrow$ iii). Let $A$ be regular open. Then $A$ is open and $A=\operatorname{int}(\operatorname{cl} A)=\operatorname{int}(\operatorname{cl}(\operatorname{int} A))$. Therefore, every regular open set is $\alpha$-open and semi-preclosed. 
iii) $\Rightarrow$ i). Let $A$ be $\alpha$-open and semi-preclosed. Then $\operatorname{int}(\operatorname{cl}(\operatorname{int} A)) \subset A \subset \operatorname{int}(\operatorname{cl}(\operatorname{int} A))$. Therefore, $A=\operatorname{int}(\operatorname{cl}(\operatorname{int} A))$ and hence $\operatorname{int}(\operatorname{cl} A)=\operatorname{int}(\operatorname{cl}(\operatorname{int}(\operatorname{cl}(\operatorname{int} A))))=\operatorname{int}(\operatorname{cl}(\operatorname{int} A))=A$. Hence $A$ is regular open.

Corolary 2. Suppose A is pre-regular sp-open. Then the following are hold:

i) If $\mathrm{A}$ is open, then $\mathrm{A}$ is regular open.

ii) If $\mathrm{A}$ is closed, then $\mathrm{A}$ is clopen.

iii) If $\mathrm{A}$ is semi-open, then $\mathrm{A}$ is regular open.

iv) If $\mathrm{A}$ is semi-closed, then $\mathrm{A}$ is $\alpha$-open and semi-preclosed.

Proof. Since $A$ is pre-regular $s p$-open, by Theorem $2.5 A=\operatorname{spclA} \cap \operatorname{int}(\operatorname{cl} A)=\operatorname{pint} A \cup \operatorname{int}(\operatorname{cl}(\operatorname{int} A))$.

i) Suppose $A$ is open. Then by Diagram, $A$ is a $q$-set and by Theorem 2.18 , we have $A$ is regular open.

ii) Suppose $A$ is closed. Now $A=\operatorname{spcl} A \cap \operatorname{int}(\operatorname{cl} A)=\operatorname{spcl} A \cap \operatorname{int} A=\operatorname{int} A$. Hence $A$ is open and hence clopen.

iii) Since every semi-open set is a $q$-set, by Theorem $2.18 \mathrm{~A}$ is regular open.

iv) Suppose $A$ is semi- closed. Then $\operatorname{int}(\operatorname{cl} A) \subseteq A$. This implies $\operatorname{int}(\operatorname{cl} A) \subset \operatorname{int} A \subset \operatorname{cl}(\operatorname{int} A)$. Hence $A$ is a $q$-set and by Theorem $2.18, A$ is $\alpha$-open and semi-preclosed.

Remark 2.17. In a partition space $(X, \tau)$, a subset $A$ of $X$ is preopen if and only if $A$ is pre-regular sp-open.

Theorem 2.18. If a space $(\mathrm{X}, \tau)$ is submaximal, then any finite intersection of pre-regular sp-open sets is pre-regular sp-open.

Proof. Let $\left\{A_{i} \mid i \in I\right\}$ be a finite family of pre-regular $s p$-open sets. Then $\left\{A_{i} \mid i \in I\right\}$ is a finite family of preopen sets. Since $X$ is submaximal, $\bigcap_{i \in I} A_{i}$ is pre open. Therefore by Theorem 2.2 (ii), $\bigcap_{i \in I} A_{i} \subseteq \operatorname{pint}\left(\operatorname{spcl}\left(\bigcap_{i \in I} A_{i}\right)\right.$. On the other hand, for each $i \in$ I, we have $\bigcap_{i \in I} A_{i} \subseteq A_{i}$ and by Theorem 2.2 (i) $\left.\operatorname{pint}\left(\operatorname{spcl}\left(\bigcap_{i \in I} A_{i}\right)\right)\right) \subseteq \operatorname{pint}\left(\operatorname{spcl} A_{i}\right)$. Since $\operatorname{pint}\left(\operatorname{spcl} A_{i}\right)=A_{i}$, we have $\left.\operatorname{pint}\left(\operatorname{spcl}\left(\bigcap_{i \in I} A_{i}\right)\right)\right) \subseteq \bigcap_{i \in I} A_{i}$. Hence $\left.\operatorname{pint}\left(\operatorname{spcl}\left(\bigcap_{i \in I} A_{i}\right)\right)\right)=\bigcap_{i \in I} A_{i}$.

Theorem 2.19. If $A$ is pre-regular sp-closed and a rare set of a space $(X, \tau)$, then $A$ is semipreopen. 
Proof. Since $A$ is pre-regular $s p$-closed, by Theorem $2.5 A=\operatorname{pcl}(\operatorname{spint} A)=\operatorname{spint} A \cup \operatorname{cl}($ int $A)$. Since $A$ is a rare set, int $A=\emptyset$. Thus $A=$ spint $A$. Hence $A$ is semi-preopen.

Recall that a space $(X, \tau)$ is said to be an extremally disconnected if the closure of every open subset of $X$ is open. Moreover, it is shown in $[7](X, \tau)$ is extremally disconnected if and only if $\mathrm{SPO}(X)=\mathrm{PO}(X)$.

Theorem 2.20. For an extremally disconnected space $(\mathrm{X}, \tau)$, the following are equivalent:

i) A is pre-regular sp-open.

ii) A is pre-regular sp-closed.

iii) $\mathrm{A}$ is pre-clopen.

iv) A is semi-preclopen.

Proof. ( $\mathfrak{i}) \Leftrightarrow$ (iii). Suppose that $A$ is pre-regular $s p$-open. Then by Theorem 2.6, $A$ is preopen and semi-preclosed. Since $X$ is extremally disconnected, $A$ is pre-clopen. Hence $A$ is pre-closed. The converse is obvious by Theorem 2.6.

(ii) $\Leftrightarrow(\mathfrak{i v})$. Let $A$ be pre-regular $s p$-closed. Then $X \backslash A$ is pre-regular $s p$-open and by $(\mathfrak{i}) \Leftrightarrow$ (iii) $X \backslash A$ is pre-clopen. Therefore, $A$ is semi-preclopen. The converse is obvious.

(iii) $\Leftrightarrow(\mathfrak{i v})$. This is obvious.

Recall that a space $(X, \tau)$ has the property $Q[10]$ if $\operatorname{int}(\operatorname{cl} A)=\operatorname{cl}(\operatorname{int} A)$ for all subset $A$ of $X$.

Theorem 2.21. Let $(\mathrm{X}, \tau)$ be a space with the property $\mathrm{Q}$. For a subset $\mathrm{A} \subseteq \mathrm{X}$, the following properties are equivalent:

i) A is pre-regular sp-open.

ii) A is pre-regular sp-closed.

iii) A is regular open.

iv) A is regular closed.

Proof. (i) $\Leftrightarrow$ (iii). By Proposition 1.3, pint $(\operatorname{spcl} A)=[A \cap \operatorname{int}(\operatorname{cl} A)] \cup \operatorname{int}(\operatorname{cl}(\operatorname{int} A))=[A \cap$ $\operatorname{int}(\operatorname{cl} A)] \cup \operatorname{int}(\operatorname{int}(\operatorname{cl} A))=\operatorname{int}(\operatorname{cl} A)$. This completes the proof.

$(\mathfrak{i i}) \Leftrightarrow(\mathfrak{i} v)$. By Proposition 1.3, $\operatorname{pcl}(\operatorname{spint} A)=[\mathrm{A} \cup \operatorname{cl}(\operatorname{int} A)] \cap \operatorname{cl}(\operatorname{int}(\operatorname{cl} A))=[A \cup \operatorname{cl}(\operatorname{int} A)] \cap$ $\operatorname{cl}(\operatorname{cl}(\operatorname{int} A))=\operatorname{cl}(\operatorname{int} A)$. This completes the proof.

(iii) $\Leftrightarrow(\mathfrak{i v})$. This is obvious. 


\section{Decompositions of complete continuity}

In this section, the notion of pre-regular $s p$-continuous functions is introduced and the decompositions of complete continuity are discussed.

Definition 5. A function $\mathrm{f}: \mathrm{X} \rightarrow \mathrm{Y}$ is said to be pre-regular sp-continuous (briefly, prspcontinuous) if $\mathrm{f}^{-1}(\mathrm{~V})$ is pre-regular sp-open in $\mathrm{X}$ for each open subset $\mathrm{V}$ of $\mathrm{Y}$.

By Theorems 2.18 and Daigram, we have the following main theorem

Theorem 3.1. For a function $\mathrm{f}: \mathrm{X} \rightarrow \mathrm{Y}$, the following properties are equivalent:

i) $\mathrm{f}$ is completely continuous.

ii) $\mathrm{f}$ is prsp-continuous and continuous.

iii) $\mathrm{f}$ is prsp-continuous and $\alpha$-continuous.

iv) $\mathrm{f}$ is prsp-continuous and semi-continuous.

v) $\mathrm{f}$ is prsp-continuous and q-continuous.

Remark 3.2. As shown by the following examples, prsp-continuity and continuity (hence $\alpha$ continuity, semi-continuity, q-continuity) are independent of each other.

Example 3.3. Let $X=\{a, b, c\}, \tau=\{\emptyset,\{a\}, X\}$ and $\sigma=\{\emptyset,\{a, b\}, X\}$. Then

i) The identity function $\mathrm{f}:(\mathrm{X}, \tau) \rightarrow(\mathrm{X}, \tau)$ is continuous but it is not prsp-continuous since $\mathbf{f}^{-1}(\{\mathbf{a}\})=\{\mathbf{a}\}$ is open but it is not pre-regular sp-open.

ii) Consider the function $\mathrm{f}:(\mathrm{X}, \sigma) \rightarrow(\mathrm{X}, \tau)$ defined by $\mathrm{f}(\mathrm{a})=\mathrm{a}, \mathrm{f}(\mathrm{b})=\mathrm{c}$ and $\mathrm{f}(\mathrm{c})=\mathrm{b}$. Then $\mathrm{f}$ is prsp-continuous but it is not q-continuous, since $\mathfrak{f}^{-1}(\{\mathbf{a}\})=\{\mathbf{a}\}$ is pre-regular sp-open but it is not a q-set in $(\mathrm{X}, \sigma)$.

\section{References}

[1] M. E. Abd El-Monsef, S. N. El-Deeb and R. A. Mahmoud, $\beta$-open and $\beta$-continuous mappings, Bull. Fac. Sci. Assiut Univ., 12 (1983), 77-90.

[2] D. Andrijević, Semi-preopen sets, Mat. Vesnik., 38 (1986), 24-32.

[3] D. Andrijević, On b-open sets, Mat. Vesnik., 48 (1996), 59-64.

[4] S. P. Arya and R. Gupta, On strongly continuous mappings, Kyungpook Math. J., 14 (1947), 131-143. 
[5] C. Chattopadhyay and C. Bandyopadhyay, On structure of $\delta$-sets, Bull. Calcutta Math. Soc., 83 (2011), 281-290.

[6] J. Dugundji, Topology, Allyn and Bacon, Boston (1966).

[7] M. Ganster and D. Andrijević, On some questions concerning semi-preopen sets, J. Inst. Math. Comp. Sci. (Math. Ser.) 1 (2) (1988), 65-75.

[8] I. A. Hasanein, M. E. Abd El- Monsef and S. N. El-Deep, $\alpha$-continuity and $\alpha$-open mappings, Acta. Math. Hungar., 41 (1983), 213-218.

[9] S. Jafari, On certain types of notions via preopen sets, Tamkang J. Math., 37 (4) (2006), 391-398.

[10] N. Levine, Semi-open sets and semi-continuity in topological spaces, Amer. Math. Monthly, 70 (1963), 36-41.

[11] A. S. Mashhour, M. E. Abd El-Monsef and S. N. El-Deep, On precontinuous and weak precontinuous mappings, Proc. Math. Phys. Soc. Egypt., 53 (1982), 47-53.

[12] O. Njåstad, On some classes of nearly open sets, Pacific J. Math. 15 (1965), 961-970.

[13] M. Stone, Applications of the theory of boolean ring to general topology, Trans. Amer. Math. Soc., 41 (1937), 374.

[14] P. Thangavelu and K. C. Rao, q-sets in topological spaces, Prog of Maths., 36 (1-2) (2002), 159-165. 